\title{
Primary Mental Health Care Part 2: Three European Primary Mental Health Care Examples as Basis for Change to the Irish System
}

\author{
Smith L,* Aherne D, O'Shea J \\ University of Limerick, Castletroy, Limerick, Ireland
}

\begin{abstract}
The successes and limitations of primary mental health care systems in three countries outside of Ireland are examined in order to inform potential change for the Irish primary mental health care system. Systems currently at work within Scotland, England, and the Netherlands are outlined, all of which employ versions of the "stepped-care" approach to primary care. It is acknowledged that Ireland is attempting to modify primary care to include the stepped-care approach. However, there are significant limitations to the current Irish system. With the Scottish, English, and Dutch systems in mind, an alternative vision of primary mental health care for Ireland is suggested.
\end{abstract}

Keywords: Mental health, Health care, Irish system

Initiatives in primary mental health care

\section{Introduction}

The current Irish primary mental health care (PMHC) service in Ireland has been examined in the first article in this series. The model, known as 'Counseling in Primary Care' (CIPC) was introduced by the Health Service Executive (HSE) in 2013 and was first evaluated in 2018. ${ }^{1}$ Shortcomings of this model include: eligibility criteria; service limitations and lengths of waiting lists; and medicalization of the whole system. Currently, in order to access CIPC services, a candidate must be aged over 18 and have a medical card. The CIPC model is a short-term service with a limit of 8 counseling sessions, and access is only available through GP referral. The most recent report on the National Evaluation Study revealed 76-80\% of service users waiting $0-4$ months and $20-24 \%$ waiting between 4-6 months. ${ }^{1}$

These shortcomings are outlined in greater detail in the first article in this series. ${ }^{2}$ In order to demonstrate alternatives to CIPC, this second article of the series considers three PMHC models out side of the Irish system. This paper commences with a review of the Scottish system, followed by a review of the system in England, and finally with a review of the Dutch system. No system stays static forever; thus, we highlight that changes have been made within the Irish service over the past few years and point to a paradigm shift that is emerging within Ireland and the UK which provides an alternative approach to the medical model. However, the changes and shifts identified are disjointed and piecemeal. With the limitations of the Irish systems and the benefits of the overseas systems in mind, this article proposes a complete reconsideration and reconfiguration of the Irish PMHC system.

\section{Scotland: Stepped-Care}

In the year 2000, Jim White, a Consultant Clinical Psychologist with the National Health Service (NHS) in Scotland, proposed a new model of PMHC which was rolled out across the Glasgow region throughout 2003. This model, described in White ${ }^{3-5}$ and Bower \& Gilbody, ${ }^{6}$ comprises two fundamental elements: firstly, that support
Quick Response Code:

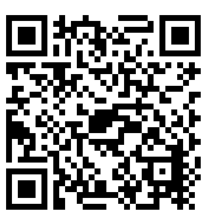

*Corresponding author: Lucy Smith, Clinical Psychology, Dip. Humanistic and Integrative Psychotherapy). Clinical Psychologist, Psychotherapist, and Head of Counselling, University of Limerick, Castletroy, Limerick, Ireland

Received: 11 May, 2021

Published: 18 June, 2021

Citation: Smith L, Aherne D, O’Shea J. Primary Mental Health Care Part 1: A Critical Review of the Irish System. J Psych Sci Res. 2021;1(2):1-8. DOI: 10.53902/JPSSR.2021.01.000509 
commences with the least intensive intervention likely to promote increased wellbeing; and secondly, that it is 'self-correcting',meaning intervention can be stepped-up to one of greater intensity as necessary without assuming non-compliance of the service user. Imperative to the stepped-care model is its accessibility to the local community and self-referral into the model rather than professional referral. These elements invite and promote help-seeking and service use, contributing to the maintenance of wellbeing in the community and mitigation of symptom development for those with difficulties. White ${ }^{5}$ suggests that the model is not only staffed by mental health practitioners but also managed by specialists in mental health, such as clinical or counselingpsychologists. ${ }^{[1]}$ Qualified psychologists are well suited to delivering a wide range of support at primary care level including therapy, awareness raising, prevention, and early intervention. Critically, however, the psychologist has competencies in assessment and formulation. Their range of skills, therefore, allow them to refer service users to the most effective interventions and services thus utilizing available resources efficiently.

Behind any stepped-care model lies a menu of interventions ranging from low to high intensity. The assessment stage which frequently lies before or at step 1 is ideally governed by the psychologist who is well trained to identify psychological needs and effectively allocate psychological interventions. Although the number of actual steps might vary slightly, Bower \& Gilbody ${ }^{6}$ note that they will be limited by two issues; a finite number of interventions that differ qualitatively regarding intensity, and the upper limit of service that can be offered by such a model. To provide an example, therefore, any model utilizing a stepped-care approach might look as follows:

[1]Henceforth, Clinical/Counselling Psychologists will be abbreviated to simply 'Psychologists'.

Step 1: Assessment/screening, existing community supports, and self-help supports (e.g., bibliotherapy, pod-casts, websites, online cognitive-behavioral programmes)

Step 2: Guided self-help (talks/groups/classes, 1-2 support sessions with a therapist)

Step 3: Brief individual therapy (1-6 sessions with a therapist)

Step 4: Longer-term individual therapy (6-20 sessions with a therapist)

Step 5: Referral to secondary care/external specialist supports

In a system with limited resources, the stepped-care model is ideal for reaching the greatest number of people possible and employing the least intensive yet effective intervention. It provides early intervention, self-help, and community based psychological support for the local population as well as immediate psychological care for low to moderate level difficulties from mental health professionals. The employment of psychologists within the model permits the provision of psychological assessment and support for those with more complex levels of care or more severe problems who may or may not require referral to secondary level care. Prevention and early intervention mitigate the development, entrench- ment, chronicity, and severity of mental health problems. As is clear from the above example, those who cannot be treated adequately within this stepped-care structure can be referred to specialist or secondary mental health care services. Such referrals can be made quickly following assessment or when PMHC supports have not been effective or sufficient.

Given that only appropriate referrals get directed to secondary care, this approach alleviates pressure on the secondary and tertiary services both in the short and the long-term. Resources at all levels of mental healthcare can therefore be employed to their greatest potential. Whilst limitations, such as high attrition rates, are associated with the stepped-care model, ${ }^{3,7,8}$ the model flexibility, self-correcting nature, and openness to improvement ${ }^{3}$ render it suitable as an evolving and adaptable primary care initiative.

\section{The United Kingdom (UK): Increasing Access to Psycho- logical Therapies (IAPT)}

'Increasing Access to Psychological Therapies' (IAPT) is the term employed for a system of care utilized within the National Health System (NHS) of the United Kingdom (UK) to provide evidenced based talk therapies for those with depression, anxiety, and co-morbid long-term physical health conditions (LTCs) or medically unexplained symptoms (MUS). ${ }^{9}$ Piloted in $2006^{10}$ and established in 2008, IAPT utilizes a stepped-care ideology (i.e., the most appropriate but least intrusive intervention is offered first) whilst operating as a 'hub and spoke' model (i.e., management and administration are centralized but 1:1 therapy is provided at local primary, community, and secondary settings). ${ }^{9}$ Referral pathways are varied (self, community, primary care, secondary care, GP) thus optimizing accessibility. Only those psychological therapies recommended by the national institute for health and care excellence (NICE) are provided by the IAPT model. Cognitive behavioral therapy (CBT) is offered for all presentations whilst interpersonal therapy (IPT) is offered as an alternative for moderate-severe depression and eye-movement desensitization and reprocessing an alternative for post-traumatic stress disorder (PTSD). ${ }^{8}$ The workforce behind IAPT consists of low-intensity and high-intensity therapists. Low intensity interventions (step 2) are provided by psychological wellbeing practitioners (PWPs) who have completed an IAPT training course whilst those providing high intensity interventions (step 3) are therapists trained in delivering CBT or IPT and who also have accreditation with their relevant professional bodies. ${ }^{9,11}$ This addition of PWPs is central to the stepped-care vision where most people may be sufficiently supported with the low-intensity interventions. $^{11}$

The most recent IAPT statistics detail that for the period April 2019 to March 2020 1,666,047 referrals were received by the service, of which $36.5 \%$ completed a course of treatment. Based on pre- and post-treatment data, $47.7 \%$ of eligible referrals moved to recovery. ${ }^{12}$ This is promising data from a large-scale, national service. However, there are some limitations and restrictions inherent within IAPT which are compromising its potential. These include: therapeutic issues, resource issues, and staff issues. Each of these are outlined below: 


\section{Therapeutic issues}

\section{Limited range of therapy}

The limited therapeutic interventions provided by the IAPT model is concerning. Guided self-help provided by the PWPs and diagnosis/symptoms specific CBT at higher intensity interventions may well be 'best practice' but the reliance on limited training and specific focus diminishes the complexity of human beings and overlooks the factors related to their social world. ${ }^{3}$ A number of reviews of psychological therapies have empirically supported the efficacy of various other therapeutic approaches beyond CBT for a wide range of psychological disorders. These include: brief dynamic therapy, emotion focused therapy, family focused therapy, and interpersonal social rhythm therapy for mood disorders; ${ }^{13}$ dynamic therapy for major depressive disorder ${ }^{14}$ irritable bowel syndrome ${ }^{15}$ and mental health problems; ${ }^{16}$ acceptance and commitment therapy for mental and physical health problems ${ }^{16}$ and integrative psychotherapy for generalized anxiety disorder. ${ }^{18}$ It is less well documented but nonetheless extremely significant that the effects of CBT have been found to decay with time following treatment completion whilst the effects of psychodynamic therapies continue to improve following completion. ${ }^{19}$ Furthermore, although himself endorsing the CBT approach as suitable to primary care, White $e^{4}$ cautions against reliance on research orientated manualized CBT, which may inhibit therapeutic initiative. This is particularly worrying in light of those PWPs with minimal training who might lack clinical competency to adapt therapy to the client. Talk therapy conducted in this way is akin to a technician 'doing' a job, thus promoting what Rizq ${ }^{20}$ has referred to as a 'perversion of care' and a 'fetishization of governance'. We suggest that limiting therapy type and practitioner skill-base renders the IAPT models inflexible, prescriptive, and unlikely to incur long-lasting change for individuals. However, a model run and staffed by fully trained psychologists and psychotherapists permits the use of clinical judgment, modification of technique, and application of integrative work as would be expected of any competent practitioner.

\section{Reliance on diagnosis, symptoms, and clinical cut off}

The IAPT model relies on diagnosis to guide treatment which in turn complies with NICE guidelines. ${ }^{21}$ Furthermore, severity of presentation and allocation to low or high-intensity intervention is determined by the nine item Patient Health Questionnaire (PHQ-9) and seven item Generalized Anxiety Disorder scale (GAD-7) clinical cut off scores (i.e., above 9 on the PHQ-9 for depression and above 7 on the GAD-7 for panic disorder, social phobia, and PTSD). The IAPT model therefore implicitly perpetuates the medical model which responds to ill-health rather than endorsing wellbeing maintenance..$^{22}$ Furthermore, by focusing exclusively on specific therapy techniques, the IAPT model ignores the three other broad areas of importance related to client outcome: extra-therapeutic factors; expectancy effects; and common factors. ${ }^{23}$ Relying heavily on specific techniques overlooks well-known variables contributing to mental wellbeing and outcome, such as resilience, optimism, and social support, ${ }^{24}$ therapist characteristics, and client-therapist interaction. ${ }^{25}$ Adherence to technique over client needs in this way has a detrimental impact on wellbeing. ${ }^{22}$
In addition, shadows have been cast in relation to IAPT's high rate of recovery. It has previously been established that scores on weekly self-report scales can drop due to repeated test administration rather than due to the effects of wellbeing improvement. ${ }^{26}$ $S \cot t^{27}$ presented independent research exploring IAPT service user recovery, employing a more comprehensive assessment (albeit also medicalized in nature), the SCID (semi-structured clinical interview for the diagnostic and statistical manual of mental disorders). Findings revealed only $9.2 \%$ of IAPT clients to fully recover from their disorder.

\section{Resource issues}

The stepped-care model proposed by White 4 places great emphasis on mental health awareness within the community, the maintenance of wellbeing via prevention and early intervention, and the provision of mental health support utilizing a variety of media (bibliotherapy, online websites and apps, blogs, and podcasts). Whilst the IAPT model has been widely received and can (supposedly) demonstrate improvement amongst service users, the heavy reliance on 1:1 intervention renders it highly resource intensive and open only to those who are motivated to attend and engage with personal work. Furthermore, although residing itself at the level of primary care, it does little to maintain large-scale public mental well-being or to de-stigmatize mental health concerns in the wider community. The outcome of research on the Scottish, steppedcare model, has already revealed that 1:1 intervention is not the most appropriate approach for everyone, particularly those from deprived areas. ${ }^{4,5}$ However, IAPT's emphasis on 1:1 work invokes a heavy financial investment from the government without consideration for White's previous findings. This heavy 1:1 focus of resources is unlikely to make any significant changes to prevent the development of mental health problems. As with the medical model, this approach intervenes only after symptoms and problems emerge rather than attempting to prevent or mitigate their development in the first place. There is no triaging of service delivery nor tailoring of therapeutic approach. This means that regardless of need or difficulty all service users are treated equally, placing undue demands on the limited resources. The primary care machine gets choked by its own self-imposed demands. The establishment of a true stepped care model which provides incrementally increased resources depending upon presentation would ease the burden on this system.

\section{Staff issues}

\section{The side-lining of psychologists}

As part of its five-year development plan to the NHS, ${ }^{27}$ IAPT is set to provide services to an additional 600,000 service users and extend services to those with long-term physical health conditions. There will also be a slightly increased focus on high-intensity interventions achieved by redistributing the IAPT workforce to $30 \%$ PWPs, $60 \%$ high intensity therapists, and 10\% clinical, health, or counselling psychologists. ${ }^{9}$ The goal is that psychologists will be available to provide expertise in LTC and MUS as well as manage complex problems and provide supervision. This appears a promising move given concerns by White ${ }^{4}$ and Rizq $^{20}$ of the relegation and dilution of applied (i.e., clinical, counselling, health) psychol- 
ogists in favor of cheaper mental health workers. However, there remains little reference to early intervention work within primary care, for which such psychologists are well suited. Furthermore, the taskforce, commissioned in 2015 which makes recommendations for change for the coming years within PMHC in the UK clearly continues to endorse the medical model and lead, stating, 'The training of GPS could also be improved to ensure they are fully supported to lead the delivery of multidisciplinary mental health support in primary care' (p.46). ${ }^{27}$ This is a disappointing addition by the taskforce and one which conflicts utterly with White's vision of placing the psychologist at the helm of PMHC.

\section{High staff turn-over}

PWPs are recruited from healthcare backgrounds and provided with one year's training and skill acquisition to provide assessments and guided self-help support with the IAPT model. However, retention of PWPs has proved a concern. ${ }^{9,27}$ Many of those applying for the role have predominantly been young, white, female graduates from psychology or related backgrounds ${ }^{28}$ who use the role as the means to an end for clinical psychology training. ${ }^{30}$ In addition, the high caseload of PWPs has contributed to burn-out ${ }^{31}$ with staff finding themselves dealing with highly complex cases for which they are insufficiently trained. The NHS9 has responded to these concerns with a set of recommendations aiming to retain staff. Such recommendations include recruiting PWPs from a wide variety of backgrounds, ensuring two-year commitment to the role, and creating development opportunities, seniority, and specialisms to promote career development. The publication of the standards of practice for the retention of $\mathrm{PWPs}^{9}$ is too recent to evaluate. It seems that the job itself is struggling to find a stable identity within mental health and that the realities of the role are conceivably stretching the skillset of the PWP.

\section{The Netherlands: Primary Care Psychology (PCP)}

Siemons ${ }^{31}$ delineates four specific psychological professions within the Dutch system: health care psychologists; psychotherapists; clinical psychologists; and clinical neuropsychologists. All these professions require undergraduate and masters level qualifications in psychology, education, or mental health to enter formal training. The former two professions take two-three-years formal training respectively following entry and are considered 'generalist' in nature. The latter two professions, considered 'specialist', are an additional four years on top of the two-year health care psychology training.

The current PMHC system in the Netherlands serves as a model for Europe and is considered 'unique' to the entire world..$^{33}$ Providing psychological care to populations within delineated geographic regions and utilizing the stepped-care model, the Dutch system is now almost 40 years old. ${ }^{33}$ Born out of the absence of brief psychological care as much as the need for master's level psychologists to find employment, psychologists began by working with family doctors to support patients who were frequent attenders to their GP but who appeared physically healthy. The role of the primary care psychologist (PCP) was created.
Today, PCPs are certified health psychologists who register with the National Organization of Primary Care Psychologists. They engage in a further year of study to provide them with the specific applicable knowledge and skill for the role, as well as to promote the necessary attitude for primary care work. This extra year also promotes and consolidates identity formation as a PCP. As stated, PCPs are referred to as 'generalists', indicating a wide skill set that allows them to assess and treat a full range of psychological difficulties throughout the lifespan. ${ }^{33}$ They work closely with family doctors, helping to review potential referrals before they are made. They provide psychological support for patients who present with psychological problems for the first time. Since 2008, eight sessions with the PCP can be reimbursed through the Dutch healthcare policy, with another four sessions frequently supplemented. ${ }^{32}$ Medical and self-referral is possible, although self-referral is on the increase $^{33,34}$ Characteristics of the service that facilitate self-referral include walk in systems and email registration rather than reliance on referral from family doctors. ${ }^{33}$ No waiting list between registration and treatment exists. Satisfaction with the service is high, personal improvement rates are at $60 \%$, drop-out rates are $18 \%$, and referral to secondary care is at $14 \%$. This stepped-care model is central to the reduction of referrals to secondary care ${ }^{35}$ with mild to moderate psychopathology successfully managed within the primary care system. ${ }^{36}$

Similar to the Scottish system of stepped-care but contrasting with the UK IAPT model, an integrated approach to health and wellbeing is provided in the Netherlands. The PCP works closely with the patient, drawing together a systemic understanding of their concerns and forming intervention plans that take a biopsychosocial approach. Treatment can, therefore, involve the main players of the patient's social life, including the family, local doctor, nurses, physiotherapist, and social workers. Individual based treatment is not the only aspect of the PCP's role, however. Unlike the CIPC model in Ireland and the IAPT model in the UK, Dutch PCPs working in primary care also place great emphasis on health promotion and early intervention work through the provision of information on various topics related to mental wellbeing and health. ${ }^{33}$ Wellbeing is not just considered the absence of illness within this model, but also the presence of quality of life and strong relationships. It is the PCP who serves as gatekeeper to care provision, utilizing observation, interview, and psychometric assessments and adjusting treatment as necessary, fitting with the stepped-care model. Their work assumes a positive psychological approach, exploring the healthy elements of the client rather than the 'sick' or 'illness' model that might be perpetuated within secondary care, or the Irish CIPC model of primary care.

The PCP, understanding the social context and biopsychosocial issues inherent to their service user, utilize the stepped-care model in a way that meets the individual's need and incorporates psychological knowledge. The stepped-care approach to depression, for example, may be as follows:

Step 1 - Watchful waiting for up to three months (given that most periods of depression remit in this time) 
Step 2 - Guided self-help and internet-based interventions

\section{Step 3 - Brief 1:1 therapy}

Step 4 - Longer 1:1 therapy and/or anti-depressant medication.36

It is noteworthy that medication is involved at step 4 rather than step 1, placing such an intervention option later in the treatment process rather than first, as is often the case in Ireland..$^{38-41}$

Collaboration and exploration of medication use in primary care, such as this, has been demonstrated a contemporary and cost-efficient method of treating common psychological presentations ${ }^{42}$ and is worth holding in mind outside of the Netherlands.

\section{Commencement of Change in Ireland}

Although advocating and operating a shared-care model of mental health service provision, the $\mathrm{HSE}^{43}$ recommended the evaluation and development of stepped-care within its framework. Three years later, a briefing paper produced by the Heads of Psychology, Ireland (HPSI) ${ }^{44}$ called for the establishment of a primary care psychology service in Ireland. HPSI recommended the following:

\section{Clinical lead from psychologists}

2. A single point of contact to the health care system

3. Supervision and training from psychologists for others providing generic psychological assessments and interventions

4. The employment of psychology graduates for the provision of low-intensity assessments and interventions (i.e., basic psychometric screening, risk-assessment, advice, and sign-posting to other services)

We present some actioned recommendations stemming from that briefing paper; the piloting of a stepped-care model in Ireland, and employment of Assistant Psychologists in a 'generalist' role within that stepped-care model. We also present the development of the HSE supported charity, 'Jigsaw', which advocates and provides community-based support for young people in Ireland and the commencement of a UK initiated paradigm shift across Ireland.

\section{Access to Psychological Therapies Ireland (APSI)}

The recommendations made by HPSI were based on a pilot model rolled out over 5 years across one area, Co. Roscommon, in Ireland. ${ }^{44}$ This model, referred to as 'Access to Psychological Services Ireland' (APSI) bears many similarities to the IAPT approach in the UK and takes a shared care approach whereby mental health professionals work as part of the wider primary care team. ${ }^{45}$ The pilot incorporated a stepped-care model, providing low-intensity psychological treatment for adults with mild to moderate mental health problems. These low-level interventions, such as guided selfhelp, online CBT, groups interventions and brief CBT, were provided by psychology graduates who assisted Clinical and Counselling Psychologists. These graduates are, therefore, referred to as 'Assistant Psychologists' (APs). Self-referral or GP/primary care staff referral made the service highly accessible, whilst next-day assessment made it highly responsive. Mental health promotion was made available through education initiatives such as community talks, newspaper articles, etc. ${ }^{44}$ Results of the pilot have proved encouraging $^{45}$ with benefits of the model reducing referrals to secondary care, permitting on-site, rapid psychological assessment, and efficient use of clinical resources. ${ }^{46}$ Follow up evaluations on access to the service, ${ }^{47}$ the effect of therapeutic alliance ${ }^{48}$ and on symptom change, user satisfaction, and referrer feedback ${ }^{49,50}$ reveal the APSI system to be effective for half of 126 service users and well considered by referrers.

The APSI studies and the HPSI briefing paper ${ }^{44}$ have served as the catalyst for the reconfiguration of PMHC in Ireland, commencing with child and adolescent services. As of 2018, the above model has commenced national roll-out. However, the APSI system lacks its own inherent step 2 and step 3 primary care interventions, relying instead on the CIPC service for 1:1 intervention, ${ }^{44,50}$ the limitations of which we have previously outlined. ${ }^{1}$ Whilst the APSI model remedies some of those problems, such as the provision of low-level psycho education programmes, it is nevertheless attempting to insert itself within existing structures and models of care that are both limited and limiting. Despite best efforts, we suggest that while the work of APSI is welcomed, the service is attempting to simply seal the widening cracks within the existing Irish PMHC, and at present is only available within one county. It is suggested by the authors that, rather than expanding APSI within the current system of PMHC, instead, a radical overhaul of service delivery in Ireland is required.

\section{The role of Assistant Psychologists (APs)}

As with all the stepped-care models described, the low-level interventions are most efficiently and economically resourced by those with generic skills rather than specialist training. Psychology graduates with undergraduate degrees and/or postgraduate master's degrees in psychology have a wealth of knowledge rendering them particularly suited to providing generic low-intensity psychological care and evidence-based treatments. Such graduates have studied a wide variety of relevant topics including but not limited to: child and adolescent development; mental health; attachment theory; identity development; cognition and distortions; behavior and behavioral modification; research methods; and statistical analysis. Within the UK, many psychology degree graduates are employed as APs or PWPs. ${ }^{29,30}$ Paid employment that recognizes existing skills and knowledge of such graduates has, until 2018, been rare in Ireland. For those wishing to gain experience within clinical psychology in Ireland, unpaid voluntary AP work has been the traditional route, with most APs having a minimum of a masters' degree in psychology. ${ }^{51}$ However, within the HPSI psychology briefing for primary care ${ }^{44}$ strong recommendations were made for the development of a paid AP grade within the grade structure for psychologists in Ireland. This recommendation has been implemented since $2017 / 2018$, with initial development of nearly 120 AP posts to work within the new PMHC teams for children and adolescents under the supervision of qualified Clinical Psychologists. This professional and financial shift within statutory Irish health care provision is long overdue and welcomed. Furthermore, it serves as a 
model and provocateur for change in community services and provides ready-made 'generalists' who are employable within a revised PMHC model. The limitation of such a role to child and adolescent PMHC services is disappointing, however.

\section{Jigsaw}

'Jigsaw' is a registered charity and national organization primarily funded by the HSE. With 13 centers located across Ireland, Jigsaw has two core aims: to provide therapeutic support to young people aged 12-25 with mild-moderate mental health presentations; and to advocate for mental health change at policy level. ${ }^{52}$ The understanding and support provided to young people assume an ecological and systemic approach rather than the traditional medicalized model of mental health. This approach moves beyond individualized therapeutic intervention, by strengthening mental health at a societal level via awareness, understanding, and skill development. Jigsaw has added some dimension to community primary mental health care, but continues to remain disjointed from the mental health care system as a whole.

\section{Power Threat Meaning Framework: A Paradigm Shift}

The current PMHC system, as previously highlighted in paper 1 of this series, ${ }^{2}$ is medicalized in nature and gate-kept by GPs. This medicalized approach to mental health breeds and perpetuates a narrative and ethos around pathology and illness, rather than recovery and wellness. The scientific method upon which medicine is organized has not proved useful for understanding, treating, or destigmatizing mental distress. Furthermore, the 'diagnoses associated with mental 'illnessesbecome confused with explanation and also promotes abdication of responsibility within individuals for change or understanding by such clinicians of the contextual issues surrounding the problems. ${ }^{53}$ Despite this, mental 'disorders' as having a biological basis and requiring medication for 'treatment' has been accepted for decades throughout the Western world. A recent paradigm shift within psychology has recognized the pathologizing of mental health and is attempting to challenge that ethos with one of recognizing distress rather than illness, particularly in relation to exploring the factors pertaining to the development and maintenance of distress. In 2013 the Division of Clinical Psychology (DCP) within the British Psychological Society (BPS) issued a position statement articulating their view that classification systems such as the Diagnostic and statistical manual of mental disorders $(D S M)^{54}$ and the International Statistical Classification of Diseases (ICD) ${ }^{55}$ are limited both conceptually and empirically in terms of understanding human distress, ${ }^{56}$ based on the rationale presented above. The DCP is not alone in their statement. Johnston \& Boyle ${ }^{5}$ point to numerous sources also seeking change regarding the understanding of mental distress, including but not limited to; the U.S. National Institute of Mental Health (NIMH) ${ }^{57}$, the Critical Psychiatry Network, ${ }^{58}$ and service users. ${ }^{59}$ The formal presentation of an alternative to the medical model of distress has recently been published by the DCP of the BPS via the 'Power, Threat, Meaning (PTM) Framework. ${ }^{53}$ Unlike the medical model which seeks to determine what is wrong with a person, the PTM framework helps facilitate discourse by asking what has happened to a person, what meaning they make of it, and what they had to do to survive. The shift in thinking has not been overlooked by psychologists in Ireland, many of whom work in the HSE. Workshops on the PTM have been held in Dublin, May 2018, (hosted by Jigsaw), in Limerick, September 2019 (hosted by the Dept. Psychology at the University of Limerick), and articles reflecting upon the framework have been published in a special issue of the 'Clinical Psychology Forum' (2019).

Ireland's mental health policy document 'A Vision for Change' 60 has recently been supplemented by an additional document, 'Sharing the Vision' (StV). ${ }^{61} \mathrm{StV}$ does make reference to positive mental health and wellbeing, however, the document fails to make detailed reference to the resourcing or structuring of relevant qualified professional teams to ensure this positive mental health strategy doesn't remain a mere vision. StV fails to address the limitations inherent with the current structure of PMHC in Ireland and makes minimal nod to the role of psychology and absolutely none to any paradigm shift, as detailed above. GPs and emergency department admissions continue to be the main access routes to mental health care in Ireland and no changes are outlined for criteria for accessing CIPC services. Thus, many of those in need of mental health supports are either unable to access services or are destined to eventually present to an emergency department when their situation has become much more complex and entrenched. ${ }^{62}$

\section{Conclusion}

Three models of PMHC outside of Ireland have been presented prior to the articulation of the limitations within the Irish PMHC system. The modifications outlined which have attempted to adapt the current system in Ireland have, to date, been sporadic and limited. In order for successful and relevant changes to be made, it is critical that Ireland looks to systems in other jurisdictions and takes into consideration overall shifts within psychology that better meet the mental health demands of the public. The three systems outlined, along with the recent paradigm shift of the PTMF, provide relevant and real markers which can be used as a guideline for a new, non-medicalized approach to adult primary mental health care in an Irish context.

Such an approach has already been developed over the past 9 years within one community setting in Ireland; a university counselling service located at the University of Limerick. The adoption of the model is presented in part three of this PMCH series and is proposed as a pilot model for systems change in Ireland.

\section{Acknowledgments}

None.

\section{Funding}

None.

\section{Conflicts of Interest}

Author declares that there is no conflict of interest.

\section{References}

1. Health Service Executive. Counselling in Primary Care Services: National Evaluation Study. Report of Phase 1. 2018. 
2. Aherne D, Smith L, O’ Shea J. Primary Mental Health Care Part 1: A Critical Review of the Irish System. Submitted to Clinical Psychology Today. 2020.

3. White J. Clinical psychology in primary care. Invited essay. Primary Care Psychiatry. 2000;6:127-136.

4. White J. Stepping up primary care. The Psychologist. 2008;21(10):844-847.

5. White J. The STEPS Model: a high volume, multi-level, multi-purpose approach to address common mental health problems. In: J. BennettLevy, H. Christensen, P. Farrand, K. Griffiths, D. Kavanagh, B. Klein, M. Lau, J. Proudfoot, D. Richards, L. Ritterband, J White, \& C Williams, editors. Oxford Guide to Low Intensity CBT Interventions: Oxford University Press; 2010.

6. Bower P, Gilbody S. Stepped care in psychological therapies: access, effectiveness and efficiency: narrative literature review. The British Journal of Psychiatry. 2005;186(1):11-17.

7. Bower P, Gilbody S. Managing common mental health disorders in primary care: conceptual models and evidence base. BMJ. 2005;330(7495):839-842.

8. Nordgreen T, Haug T, Ost LG, et al. Stepped-care versus direct faceto-face cognitive behaviour therapy for social anxiety disorder and panic disorder: a randomised effectiveness trial. Behavior Therapy. 2016;47(2):166-183.

9. National Health Service (NHS). The improving access to psychological therapies manual. The National Collaborating Centre for Mental Health. 2018.

10. Clark DM, Layard R, Smithies R, et al. Improving access to psychological therapy: Initial evaluation of two UK demonstration sites. Behaviour research and therapy. 2009;47(11):910-920.

11. Gyani A, Shafran R, Layard R, et al. Enhancing Enhancing recovery rates in IAPT services: lessons from analysis of the year one data. London: UK. 2011.

12. National Health Service (NHS). Psychological Therapies: reports on the use of IAPT services, England March 2020 Final including reports on the IAPT pilots and Quarter 4 2019-20 data. 2020.

13. Hollon SD, Ponniah K. A review of empirically supported psychological therapies for mood disorders in adults. Depression and anxiety. 2010;27(10):891-932.

14. Connolly Gibbons MB; Gallop R, Thompson D. Comparative effectiveness of cognitive therapy and dynamic psychotherapy for major depressive disorder in a community mental health setting: a randomised clinical noninferiority trial. Journal of the American Medical Association. 2016;73(9):904-911.

15. Laird KT, Tanner Smith EE, Russell AC, et al. Comparative Efficacy of psychological therapies for improving mental health and daily functioning in irritable bowel syndrome: a systematic review and metaanalysis. Clinical Psychology Review. 2017;51:142-152.

16. Steinert C, Munder T, Rabung S, et al. Psychodynamic therapy: as efficacious as other empirically supported treatments? A meta-analysis testing equivalence of outcomes. The American Journal of Psychiatry. 2017;174(10):943-953.

17. A-tjak JG, Davis ML, Morina N, et al. A meta-analysis of the efficacy of acceptance and commitment therapy for clinically relevant mental and physical health problems. Psychotherapy and psychosomatics. 2015;84(1):30-36.

18. Orvati Aziz M, Abolghasem Mehrinejad S, Hashemian K, et al. Integrative therapy (short-term psychodynamic psychotherapy and \& cognitive behavioural therapy) in the treatment of generalised anxiety disorder: A randomised controlled trial. Complementary Therapies in Clinical Practice. 2020;39:101-122.
19. Schedler J. The efficacy of psychodynamic psychotherapy. American Psychologist. 2010;65(2):98-109.

20. Rizq R. The perversion of care: psychological therapies in a time of IAPT. Psychodynamic practice: Individuals, Groups, and Organisations. 2012;18(1):7-24.

21. Gyani A, ShafranR, Layard R, et al. Enhancing recovery rates in IAPT services: lessons from analysis of the year one data. London: UK. 2011.

22. Watson J. The Disorder!: Challenging the culture of psychiatric diagnosis. Wyastone Leys, Monmouth, UK: PCCS Books; 2019.

23. Prati G, Pietrantoni L. Optimism, social support, and coping strategies as factors contributing to posttraumatic growth: a meta-analysis. Journal of Loss and Trauma. 2009;14(5):364-388.

24. Lambert MJ, Barley DE. Research summary on the therapeutic relationship and psychotherapy outcome. Psychotherapy Theory, Research, \& Practice. 2001;38(4):357-361.

25. Ackerman SJ, Hilsenroth MJ. A review of therapist characteristics and techniques positively impacting the therapeutic alliance. Clinical psychology review. 2003;23(1):1-33.

26. Longwell BT, Truaz P. The differential effects of weekly, monthly and bimonthly administrations of the Beck Depression Inventory II: psychometric properties and clinical implications. Behavior Therapy. 2015;36:265-275

27. Scott MJ. Improving access to psychological therapies (IAPT)-The need for radical reform. Journal of Health Psychology. 2018;1-12.

28. Mental Health Taskforce. The five year forward view for mental health: a report from the independent mental health taskforce to the NHS. England; 2016.

29. Health Education England. Widening participation to psychological wellbeing practitioner training: Project report to the health education England. (Draft Report). 2017.

30. Moreea 0. Where have all the psychological wellbeing practitioners gone? Northern IAPT practice research network; 2015.

31. Steel C, Macdonald J, Schroder T. Exhausted but not cynical: burnout in therapists working within Improving Access to Psychological Therapy Services. Journal of Mental Health. 2015;24(1):33-37.

32. Siemons J. Psychologists in health care in the Netherlands. Federatie van Gezondheids zorgpsychologen; 2014.

33. LVE. Annual report of the National Organisation of Primary Care Psychologists. Amsterdam. In: Derksen J, editor. Primary care psychologists in the Netherlands: 30 years of experience. Professional Psychology: Research and Practice. 2007;40(5):493-501.

34. Meeuwissen JAC, Van der Feltz Cornelis CM, Van Marwijk HWJ. Et al. A stepped-care programme for depression management: an uncontrolled pre-post study in primary and secondary care in The Netherlands. International Journal of Integrated Care. 2008;8(1):e05.

35. Verhaak PF, Kamsma H, Van der Niet A. Mental health treatment provided by primary care psychologists in the Netherlands. Psychiatric Services. 2013;64(1):94-97.

36. Van Straten A, Seekles W, Van't Veer-Tazelaar NJ, et al. Stepped-care for depression in primary care: what should be offered and how? Medical Journal of Australia. 2010;192(11 Suppl.):S36.

37. Meijer WE, Heerdink ER, Leufkens HG, et al. Incidence and determinants of long-term use of antidepressants. European Journal of Clinical Pharmacology. 2004;60(1):57-61.

38. Johnson CF, Macdonald HJ, Atkinson P, et al. Reviewing long-term antidepressants can reduce drug burden: a prospective observational cohort study. British Journal of General Practice. 2012;(604):e773-779. 
39. Petty DR, House A, Knapp P, et al. Prevalence, duration and indications for prescribing of antidepressants in primary care. Age Ageing. 2006;35(5):523-526.

40. Mojtabai R, Olfson M. National trends in long-term use of antidepressant medications: results from the US national health and nutrition examination survey. Journal of Clinical Psychiatry. 2014;75(2):169-177.

41. Bosman RC, Huijbregts KM, Verhaak PF, et al. Long-term antidepressant use: a qualitative study on perspectives of patients and GPs in primary care. British Journal of General Practice. 2016;66(651):e708-e719.

42. Health Service Executive. Health Service Executive Primary Care and Mental Health Group. Advancing the shared care approach between primary care and specialist mental health services: a guidance paper. Naas: Office of the Assistant National Director Mental Health; 2012.

43. Heads of Psychology Services Ireland (HPSI). Psychology briefing paper for the HSE primary care division. Laois/Offaly: HSE; 2015.

44. McHugh P, Brennan J, GalliganN, et al. Evaluation of a primary care adult mental health service: Year 2. Mental Health in Family Medicine. 2013;10(1):53-59.

45. McHughP, ByrneM. Profile of the shared care activities of mental health teams in Ireland. Mental Health in Primary Care Implementation Subgroup. 2013.

46. McHughP, Martin N, Hennessy M, et al. An evaluation of access to psychological services Ireland: year one outcomes. Irish Journal of Psychological Medicine. 2016;33(4):225-233.

47. McHugh P. The effect of therapeutic alliance on psychotherapy outcomes. Clinical Psychology Today. 2018;1(2):p.32.

48. Walshe C, Walsh Z, Clogher L, et al. An evaluation of an Irish steppedcare, assistant psychologist-delivered psychological service: APSI: years two and three. Clinical Psychology Today. 2019;3(1):35-51.

49. Collins P, Walsh Z, WalshA, et al. A 360 degree evaluation of steppedcare psychotherapy: APSI yrs 4-5. Mental Health Review Journal. 2020.

50. Kelly J, SammonN, Byrne M. APSI: a proposed integrative model for suicide prevention. Irish Journal of Psychological Medicine. 2014;31(3):203-212.

51. Hughes A, Campbell M, Byrne M. Profiling Assistant Psychologist experiences in Ireland and the United Kingdom. The Irish Psychologist. 2015;41(5):107-112.

52. Jigsaw. Our strategy: 2018-2020. 2018.
53. Johnstone L, Boyle M, Cromby J, et al. Power Threat Meaning Framework: Towards the identification of patterns in emotional distress, unusual experiences and troubled or troubling behaviour, as an alternative to functional psychiatric diagnosis. Leicester: British Psychological Society; 2018.

54. MLA. Diagnostic And Statistical Manual of Mental Disorders: DSM-5. Arlington, VA: American Psychiatric Association, 2013.

55. World Health Organization. ICD-10: international statistical classification of diseases and related health problems: tenth revision, 2nd ed. World Health Organization.World Health Organization. ([2004)". ICD-10: international statistical classification of diseases and related health problems : tenth revision, $2^{\text {nd }}$ ed. World Health Organization.

56. Division of Clinical Psychology. Classification of behaviour and experience in relation to functional psychiatric diagnosis: Time for a paradigm shift. Leicester: British Psychological Society; 2013.

57. InselT. Director's blog: Transforming diagnosis. In: Johnstone L \& Boyle M, Cromby J, Dillon J, Harper D, Kinderman P, Longden E, Pilgrim D, Read J, et al. 2018. The Power Threat Meaning Framework: Towards the identification of patterns in emotional distress, unusual experiences and troubled or troubling behaviour, as an alternative to functional psychiatric diagnosis. Leicester: British Psychological Society.

58. Critical Psychiatry Network. Statement by the Critical Psychiatry Network on the new DSM-5. 2013. In: Johnstone L, Boyle M, Cromby J, Dillon J, Harper D, Kinderman P, Longden E, Pilgrim D, Read J. The Power Threat Meaning Framework: Towards the identification of patterns in emotional distress, unusual experiences and troubled or troubling behaviour, as an alternative to functional psychiatric diagnosis. Leicester: British Psychological Society. 2018.

59. Hearing Voices Network. Position Statement on DSM-5 and Psychiatric Diagnosis. In: Johnstone L, Boyle M, Cromby J, Dillon, J, Harper D, Kinderman P, Longden E, Pilgrim D, Read J. The Power Threat Meaning Framework: Towards the identification of patterns in emotional distress, unusual experiences and troubled or troubling behaviour, as an alternative to functional psychiatric diagnosis. Leicester: British Psychological Society. 2018.

60. Irish Department of Health \& Children. A vision for change: report of the expert group on mental health policy. Dublin: Stationary Office; 2006.

61. Irish Department of Health. Sharing the Vision: A Mental Health Policy for Everyone. Dublin: Stationary Office; 2020.

62. Bebbington PE, Meltzer H, Brugha TS, et al. Unequal access and unmet need: neurotic disorders and the use of primary care services. Psychol Med. 2000;30(6):1359-1367. 